\title{
Computer simulation of pressure welding with a shear of samples from dissimilar nickel-based superalloys
}

\author{
A. Kh. Akhunova ${ }^{\dagger}$, V. A. Valitov, E. V. Galieva \\ †akhunova.a.kh@gmail.com
}

Institute for Metals Superplasticity Problems RAS, 39 S. Khalturin St., Ufa, 450001, Russia

\begin{abstract}
A stress-strain state computer simulation of samples imitating bimetallic "disk-shaft" type parts for a gas turbine engine from dissimilar heat-resistant nickel-based superalloys during pressure welding with a shear was carried out. The simulation was performed in a two-dimensional formulation (axisymmetric problem) using the DEFORM-2D. Two shaft motion schemes, embedding of a shaft into a disk and a combination of the embedding and rotation of the shaft, were considered. To determine the influence of the microstructure of samples on the plastic deformation process in the joint zone, two combinations of nickel-based superalloys were considered: the material for the shaft in all cases was EK79 (fine-grained microstructure, $7 \mu \mathrm{m}$ ), the material for the disk was EP741NP (coarse-grained microstructure, $60 \mu \mathrm{m}$ ) or EP975 (fine-grained microstructure, $8 \mu \mathrm{m}$ ). The constitutive relations for the superalloys under study were introduced into the program as the experimental stress-strain curves obtained from uniaxial compression of cylindrical samples. Computer simulation results showed that to improve the joint quality it was preferable to use pressure welding with the embedding and rotation of the shaft. In this case two-component shear deformation was provided that lead to a relative shift of the surfaces to weld and an improvement of the conditions for the formation of physical contact. It has been established that to obtain a solid phase joint between the shaft and the disk, it is preferred to use the EK79 (shaft) and EP975 (disk) superalloys, since in this case the summarized normal compressive stresses and shear deformations are provided which leads to an increase of the joint quality.
\end{abstract}

Keywords: finite-element modeling, pressure welding, superplasticity, nickel-based superalloy.

УДК: 621.791 .18

\section{Компьютерное моделирование сварки давлением со сдвигом образцов из разноименных никелевых сплавов}

\author{
Ахунова А.Х. ${ }^{\dagger}$, Валитов В. А., Галиева Э. В. \\ Институт проблем сверхпластичности металлов РАН, ул. С. Халтурина, 39, Уфа, 450001, Россия
}

Проведено компьютерное моделирование напряженно-деформированного состояния в образцах, имитирующих составные части биметаллической детали газотурбинного двигателя типа «диск-вал» из разнородных жаропрочных никелевых сплавов, при их сварке давлением (СД) со сдвигом. Моделирование проводили в двумерной постановке (осесимметричная задача) с помощью пакета прикладных программ DEFORM-2D. Для определения влияния схемы СД на пластическую деформацию в зоне соединения было рассмотрено две схемы движения вала: внедрение вала в диск, и комбинация внедрения и вращения вала. Для определения особенностей влияния микроструктуры образцов на процесс протекания пластической деформации в зоне соединения свариваемых деталей рассматривались два сочетания разноименных никелевых сплавов: материалом для вала во всех случаях был сплав ЭК79 (мелкозернистая микроструктура, 7 мкм), для диска использовались сплавы ЭП741НП (крупнозернистая микроструктура, 60 мкм) и ЭП975 (мелкозернистая микроструктура, 8 мкм). Определяющие соотношения для изучаемых сплавов вводились в программу в виде экспериментальных кривых «напряжение-деформация», полученных при испытании образцов на одноосное сжатие цилиндрических образцов в условиях, соответствующих процессу сварки. Результаты компьютерного моделирования показали, что для повышения качества сварного соединения вала и диска предпочтительно использовать сварку давлением, осуществляемую при комбинации внедрения с одновременным вращением вала, поскольку в этом случае обеспечивается двухкомпонентная сдвиговая деформация, которая приводит к относительному сдвигу свариваемых поверхностей и улучшению условий образования физического контакта. Установлено, что для создания неразъёмного соединения вала и диска предпочтительно использовать 
сочетание сплавов ЭК79 (вал) и ЭП975 (диск), поскольку в этом случае обеспечивается эффективное суммарное воздействие нормальных сжимающих напряжений и сдвиговых деформаций, что приводит к повышению качества твердофазного соединения.

Ключевые слова: конечно-элементное моделирование, сварка давлением, сверхпластичность, никелевый сплав.

\section{1. Введение}

Малые газотурбинные двигатели (ГТД) находят широкое применение не только в авиации, но в области малой энергетики [1-2]. Ротор двигателя малого ГТД представляет собой специально спрофилированный диск, на периферии которых в замковой части закреплены рабочие лопатки. Крутящий момент к диску передается от вала за счет их соединения между собой. Из-за сравнительно небольших размеров, диск и вал ротора малого ГТД могут изготавливаться как одно целое методами горячего деформирования с последующей токарной обработкой [2-3]. Однако значительная трудоемкость и энергоемкость данной технологии делает предпочтительным изготовление таких деталей по отдельности с последующим их соединением.

Для соединения деталей конструкций при производстве ГТД без использования крепежных элементов используют различные методы сварки [4-8], наиболее перспективным среди которых является сварка давлением (СД) в условиях сверхпластичности [9-11]. Одним из основных достоинств СД является возможность сохранения структуры свариваемых заготовок, что важно для изделий, эксплуатируемых в условиях высоких температур и изготовленных, соответственно, из жаропрочных никелевых сплавов, которые широко используются для производства частей ротора малых ГТД [4].

Известно, что на качество твердофазного соединения (ТФС) при СД влияние оказывает не только рельеф свариваемых поверхностей [12-13], геометрия соединяемых деталей [14], температурно-скоростные условия, с помощью которых осуществляется сварка, исходная микроструктура заготовок, но и напряженнодеформированное состояние в заготовке вблизи контактных поверхностей [15-16]. Применяемая обычно при СД деформация по схеме одноосного сжатия может приводить к возникновению в области ТФС соединяемых материалов неоднородного напряженнодеформированного состояния вследствие возникновения зон затрудненной деформации. Для преодоления таких зон в заготовках приходится увеличивать усилия деформирования и соответственно степень деформации, что приводит к значительному скоплению дислокаций в зоне ТФС, а также к неизбежному деформационному упрочнению материала заготовок, что ведет к возникновению пор. В качестве метода, позволяющего избежать подобных недостатков, может быть использована комбинированная схема СД, в которой присутствует сдвиговая компонента деформации. В этом случае обеспечивается максимальная сдвиговая пластическая деформация, которая достигает высоких значений в очень тонком слое вблизи контактной поверхности соединяемых деталей и в основном не затрагивает остальной объем материала, что очень важно для таких ответственных деталей авиадвигателя, как диск и вал.

В данной работе рассматриваются методы получения сварного соединения при СД, осуществляемой воздействием деформирующего инструмента на диск и/или вал, находящийся в контакте с диском. В качестве материала для диска были выбраны высоколегированные никелевые сплавы с объемным содержанием (55-60\%) упрочняющей фазы: деформируемый ЭП975 и гранульный ЭП741НП для диска, и среднелегированный $(40 \%)$ и более технологичный деформируемый сплав ЭК79 - для вала.

\section{2. Методика компьютерного моделирования}

Компьютерное моделирование проводили в двумерной постановке (осесимметричная задача) с помощью пакета прикладных программ DEFORM-2D. Рассматривались модельные биметаллические детали типа «диск-вал». Вал представлял собой цилиндр диаметром 12 мм и высотой 12 мм, сопряженный с усеченным конусом высотой 20 мм. Угол а между образующими цилиндра и усеченного конуса составлял $1^{\circ}$. Отверстие в диске имело сужение с углом наклона на внутренней стенке, соответствующим конусности вала. Общая высота диска - 14 мм, а диаметр 28 мм. Конечно-элементная модель вала и диска приведена на Рис. 1а. При расчете рассматривалась половина системы с учетом плоскости симметрии на левой границе расчетного блока.

Коэффициент трения по Зибелю $k$ на всех контактных поверхностях во всех случаях принимался равным 0.3. Температура испытаний составляла $1100^{\circ} \mathrm{C}$.

Для определения влияния схемы СД образцов на пластическую деформацию в зоне их соединения были рассмотрены две схемы движения вала, задаваемые посредством верхней траверсы: 1) Схема 1 - внедрение вала в диск путем задания смещения верхней траверсы на 2.5 мм со скоростью перемещения 1.9 мм/мин, что соответствует начальной скорости деформации цилиндрической части вала $\left.10^{-3} \mathrm{c}^{-1} ; 2\right)$ Схема 2 комбинация внедрения и вращения вала. Вал внедрялся в диск путем смещения верхней траверсы на 2.5 мм со скоростью перемещения 1.9 мм/мин с одновременным заданием вращения за верхнюю часть вала со скоростью вращения 3 об/мин.

Деформирующие инструменты (траверсы) принимались абсолютно жесткими, деформируемые тела (диск и вал) имели свойства упруго-пластичных материалов. Для определения особенностей влияния микроструктуры образцов на процесс протекания пластической деформации в зоне соединения свариваемых деталей рассматривались два сочетания разноименных никелевых сплавов. В качестве материала для вала во всех случаях был рассмотрен сплав ЭК79 с мелкозернистой (МЗ) 
структурой типа микродуплекс с размером зерен $\gamma$-фазы и некогерентных частиц $\gamma^{\prime}$-фазы менее 7 мкм. В качестве материала для диска рассматривались два сплава: высоколегированный деформируемый сплав ЭП975 с мелкозернистой (М3) структурой типа микродуплекс с размером зерен $\gamma$-фазы и некогерентных частиц $\gamma^{\prime}$-фазы менее 8 мкм и гранульный сплав ЭП741НП с крупнозернистой (КЗ) микроструктурой со средним размером зерен $\gamma$-фазы 60 мкм.

Отметим, что при температуре $1100^{\circ} \mathrm{C}$ и скорости деформации $10^{-3} \mathrm{c}^{-1}$ сплавы ЭК79 и ЭП975 с мелкозернистой структурой типа микродуплекс демонстрируют сверхпластические свойства. Определяющие соотношения для изучаемых сплавов вводились в систему DEFORM-2D согласно экспериментальным данным в виде схематических кривых «напряжение-деформация». Была использована упруго-пластическая модель материала (идеальная пластичность), в которой напряжение течения не зависит от деформации и в указанном выше температурно-скоростном режиме составляет 50 МПа для ЭП975 (М3), 30 МПа для ЭК79 (МЗ) и 150 МПа для ЭП741НП (КЗ).

Для сравнительного анализа было проведено моделирование СД цилиндрических образцов с начальной скоростью деформации $10^{-3} \mathrm{c}^{-1}$ при температуре $1100^{\circ} \mathrm{C}$. Диаметр образцов составлял 16 мм, высота верхнего и нижнего образцов - 16 и 20 мм, соответственно. В качестве материала для нижнего образца были выбраны сплавы ЭП741НП и ЭП975, для верхнего образца во всех случаях - сплав ЭК79. Конечно-элементная модель образцов приведена на Рис. 1b.

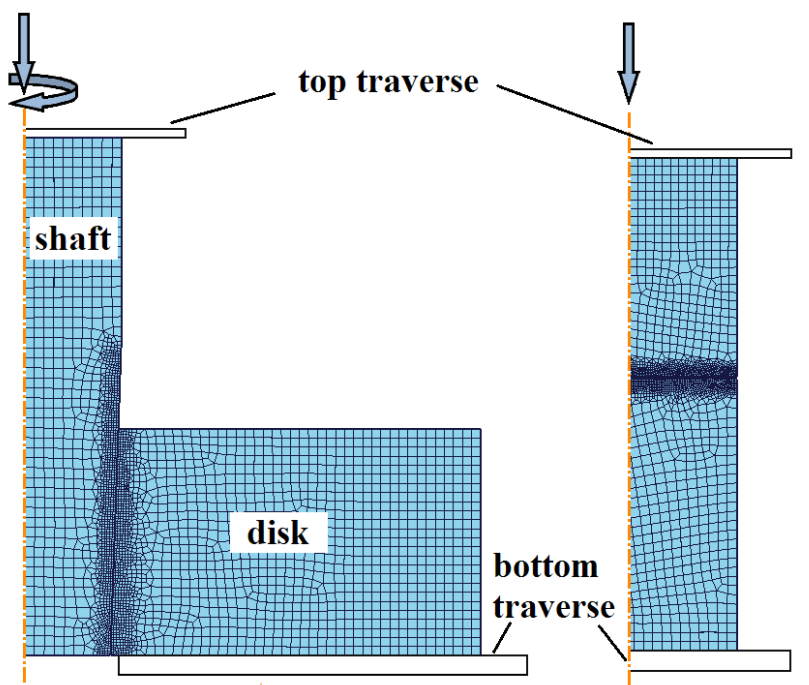

a

$\mathrm{b}$

Pиc. 1. (Color online) Конечно-элементные модели образцов, имитирующих биметаллические детали газотурбинного двигателя «диск-вал» (a), и цилиндрических образцов (b). Более тонкое разбиение сетки на элементы задавалось в зоне контактного взаимодействия заготовок.

Fig. 1. (Color online) The finite element models of samples imitating bimetallic "disk-shaft" type parts for a gas turbine engine (a) and cylindrical samples (b). Finer grids of elements were set in the contact interaction zones of workpieces.

\section{3. Результаты моделирования}

При анализе результатов компьютерного моделирования рассматривалось распределение сдвиговых компонент деформации, поскольку именно они в большей степени активизируют пластическую деформацию на контактных поверхностях.

Рассмотрим СД цилиндрических образцов по схеме одноосного сжатия, т.е. простой осадки, результаты моделирования которой представлены на Рис. 2. Картины распределения сдвиговых деформаций приведены при степени деформации верхнего образца из сплава ЭК79, равной 4\%. Отметим, что указанная степень деформации эквивалентна степени деформации, достигаемой в вале при моделировании СД в заданных условиях и рассмотренных схемах сварки. Деформация при осадке протекает неоднородно вследствие наличия так называемых застойных зон. Это объясняется тем, что сдвиговая компонента деформации в центре заготовок мала, при этом на контактной поверхности нижнего, более прочного образца, она практически отсутствует. Помимо этого, протяженность застойных зон также зависит от исходной микроструктуры. Так, в случае СД заготовок из ЭК79 и ЭП741НП, величина застойной зоны меньше, чем при СД заготовок из ЭК79 и ЭП975, т. к. в последнем случае пластичность сплава ЭП975 (М3) существенно больше, чем ЭП741НП (КЗ), но примерно одинакова со сплавом ЭК79 (МЗ).

В то же время, использование СД со сдвиговой компонентой деформации, как в случае сварки вала и диска, приводит к увеличению числа активных центров как на поверхности заготовки диска, так и на поверхности заготовки вала. Кроме того, пластическое течение материала вдоль поверхности контакта позволяет залечивать все несплошности, которые могут образоваться в процессе создания физического контакта.

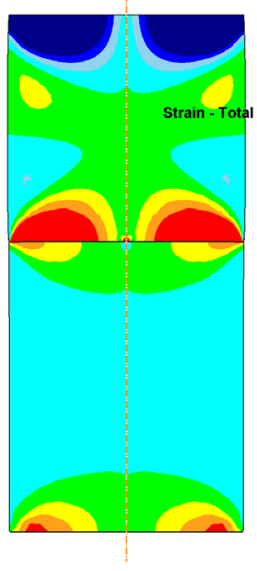

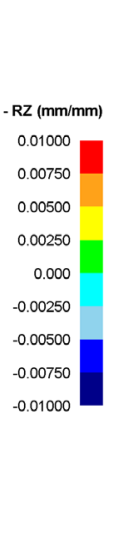

Рис. 2. (Color online) Распределение сдвиговых деформаций при СД цилиндрических образцов: верхний образец из сплава ЭК79, нижний образец - сплавы ЭП741НП (a) и ЭП975 (b).

Fig. 2. (Color online) The distribution of shear strain during pressure welding of cylindrical samples: the upper specimen is made of EK79 superalloy, the lower specimen of EP741NP (a) and EP975 (b) superalloys. 
Отметим, что при СД по Схеме 1 (внедрение вала в диск) можно говорить об однокомпонентном сдвиге, поскольку в этом случае преобладает одна компонента сдвиговой деформации $\varepsilon_{R Z}$. При СД по Схеме 2 (комбинация внедрения с одновременным вращением вала) все три сдвиговые компоненты $\varepsilon_{R Z}, \varepsilon_{R \theta}$ и $\varepsilon_{z \theta}$ становятся ненулевыми. Но если $\varepsilon_{R Z}$ и $\varepsilon_{R \theta}-$ это сдвиговые компоненты деформации, которые вызывают активизацию движения контактных поверхностей друг относительно друга, то $\varepsilon_{z \theta}$ приводит к совместной сдвиговой деформации контактирующих поверхностей. Таким образом, в этом случае можно говорить о двухкомпонентном сдвиге.

Результаты моделирования позволили установить, что значения эффективных напряжений в процессе сварки вала и диска для всех сочетаний материалов и всех рассмотренных схем сварки не превышала предела текучести в пределах погрешности. Это означает, что физический контакт формируется только за счет микропластической деформации в приконтактных областях без значительного деформирования деталей в объеме. При этом максимальные значения напряжения больше при сварке вала с диском из сплава ЭП741НП (Рис. S1, дополнительный материал), чем при сварке с диском из ЭП975 (Рис. S2, дополнительный материал), и такая закономерность наблюдается как для Схемы 1, так и для Схемы 2.

На Рис. 3 и 4 приведены эпюры распределения нормальных радиальных напряжений, полученных при СД вала с диском из ЭП741НП и ЭП975, соответственно. Известно [6,7], что сжимающие напряжения в зоне ТФС могут оказывать благоприятное влияние на образование физического контакта между заготовками. Результаты моделирования показали, что зоны со сжимающими радиальными напряжениями возникают в центральных частях заготовок вала и диска в области их контакта, при этом они более равномерно распределены при СД диска из сплава ЭП975, чем при СД диска из ЭП741НП.

По картинам распределения сдвиговых деформаций при СД по Схеме 1 можно отметить, что в случае сварки вала с диском из ЭП741НП максимальные сдвиговые

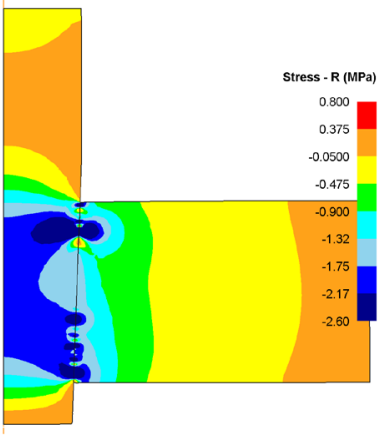

a

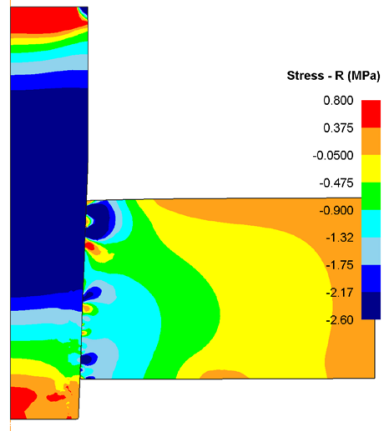

b
Pис. 3. (Color online) Распределение радиальных напряжений в вале (ЭК79) и диске (ЭП741НП) при СД по Схеме 1 (а) и Схеме 2 (b).

Fig. 3. (Color online) The distribution of radial stress in the shaft (EK79) and disk (EP741NP) when pressure welding with a shear according to Scheme 1 (a) and Scheme 2 (b). деформации формируются вблизи контактной поверхности диска (Рис. 5a), а в случае сварки с диском из ЭП975 - в основном вблизи контактной поверхности вала (Рис. 6 a)

При СД по Схеме 2 (комбинация внедрения с одновременным вращением вала) максимальные значения $\varepsilon_{R Z}$ формируются как на контактной поверхности диска, так и на контактной поверхности вала. При этом область распределения максимальных $\varepsilon_{R Z}$ становится более однородной и протяжённой, чем при тех же условиях сварки по Схеме 1. Это особенно хорошо видно по картинам распределения $\varepsilon_{R Z}$, полученным при СД вала с диском из ЭП975 (Рис. 6b), по сравнению с $\varepsilon_{R Z}$, полученным в случае диска из ЭП741НП (Рис. 5b).

Как было сказано выше, при СД по Схеме 2 обеспечивается двухкомпонентная сдвиговая деформация $\varepsilon_{R Z}$ и $\varepsilon_{R \theta}$, стимулирующая относительный сдвиг свариваемых поверхностей, что улучшает качество сварного соединения. Как видно из результатов моделирования, при СД по Схеме 2 увеличивается протяжённость областей с максимальными сдвиговыми деформациями, и достигается более равномерное их распределение в зоне контактных поверхностей.

В результате силового воздействия на соединяемые детали в приконтактных зонах возникают внутренние напряжения. Очевидно, что сжимающие напряжения в зоне ТФС могут оказаться полезными и привести к повышению прочности и надежности соединения за счет улучшения условий для образования физического контакта. Однако следует учитывать, что большие напряжения нежелательны, т.к. их величина может превышать прочность материала и привести к его нежелательной деформации.

По результатам моделирования можно заключить, что при сварке вала и диска из ЭП975 материалы деталей испытывают меньшие напряжения и деформации, чем при идентичных условиях сварки вала и диска из ЭП741НП. Максимальные значения эффективных напряжений при СД диска из ЭП741НП на порядок больше, чем при СД диска из ЭП975 - 10 МПа против 2 МПа для обеих схем. При этом при СД диска

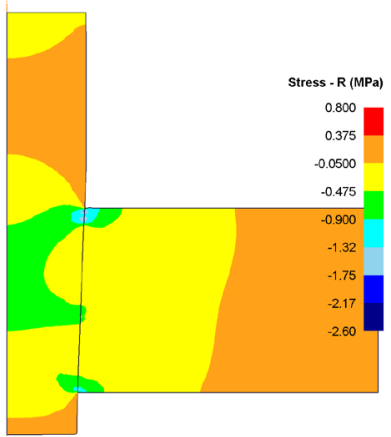

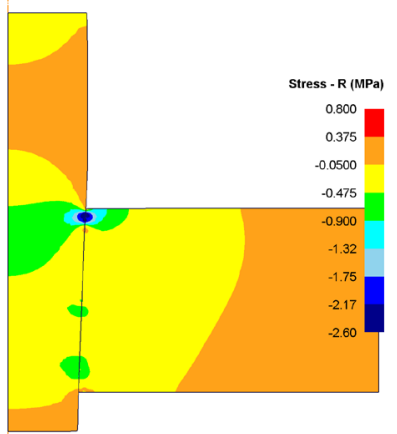

Pис. 4. (Color online) Распределение радиальных напряжений в вале (ЭК79) и диске (ЭП975) при СД по Схеме 1 (а) и Схеме 2 (b).

Fig. 4. (Color online) The distribution of radial stress in the shaft (EK79) and disk (EP975) when pressure welding with a shear according to Scheme 1 (a) and Scheme 2 (b). 


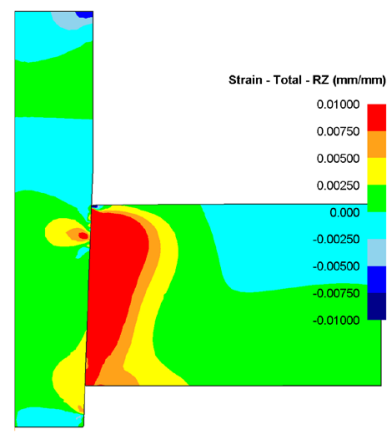

a

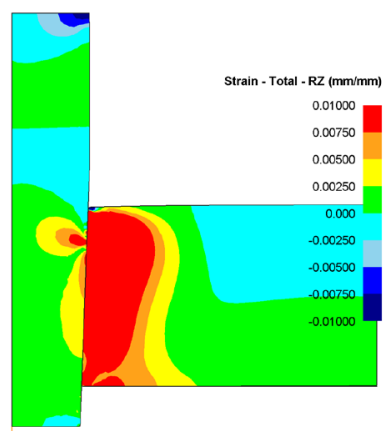

$\mathrm{b}$
Рис. 5. (Color online) Распределение сдвиговых деформаций в вале (ЭК79) и диске (ЭП741НП) при СД по Схеме 1 (a) и Схеме 2 (b).

Fig. 5. (Color online) The distribution of shear strain in the shaft (EK79) and disk (EP741NP) when pressure welding with a shear according to Scheme 1 (a) and Scheme 2 (b).

из ЭП975 в деталях достигается более равномерное распределение положительно влияющих на качество сварного соединения сжимающих нормальных напряжений, однако их максимальное значение меньше, чем при идентичных условиях сварки диска из ЭП741НП.

\section{4. Выводы}

Таким образом, можно сделать следующие выводы:

1. Для повышения качества сварного соединения вала и диска предпочтительно использовать сварку давлением, осуществляемую при комбинации внедрения с одновременным вращением вала, поскольку в этом случае обеспечивается двухкомпонентная сдвиговая деформация, которая приводит к относительному сдвигу свариваемых поверхностей и улучшению условий образования физического контакта.

2. По результатам моделирования установлено, что зоны максимальных нормальных сжимающих напряжений и сдвиговых компонент деформации вблизи контактных поверхностей более равномерно распределены при сварке вала с диском из ЭП975 (МЗ), чем при идентичных условиях сварки с диском из ЭП741НП (КЗ).

3. Для создания неразъёмного соединения вала и диска предпочтительно использовать сочетание сплавов ЭК79 (вал) и ЭП975 (диск), поскольку в этом случае обеспечивается эффективное суммарное воздействие нормальных сжимающих напряжений и сдвиговых деформаций, что приводит к повышению качества твердофазного соединения.

Дополнительный материал/Supplementary material. Электронная версия статьи содержит дополнительный материал, доступный безвозмездно на сайте журнала (lettersonmaterials.com). / The online version of this paper contains supplementary material available free of charge at the journal's Web site (lettersonmaterials.com)

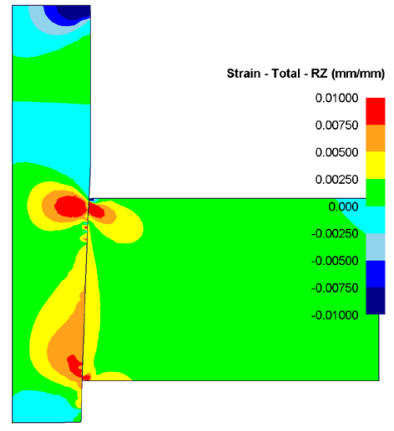

a

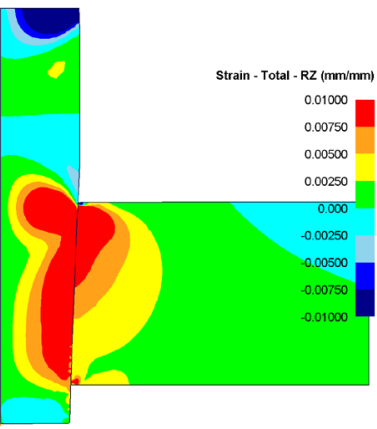

$\mathrm{b}$
Pис. 6. (Color online) Распределение сдвиговых деформаций в вале (ЭК79) и диске (ЭП975) при СД по Схеме 1 (а) и Схеме 2 (b).

Fig. 6. (Color online) The distribution of shear strain in the shaft (EK79) and disk (EP975) when pressure welding with a shear according to Scheme 1 (a) and Scheme 2 (b).

Благодарности / Acknowledgements. Моделирование и разработка схемы сварки давлением проведено при поддержке гранта РНФ №18-19-00685, а экспериментальные данные для моделирования получены по госзаданию № АAАA-A17-117041310215-4. / The computer modeling and development of pressure welding scheme was supported by the Russian Science Foundation (Grant No. 18-19-00685). A mechanical properties part was supported by the IMSP RAS State assignment No. AAAA-A17-117041310215-4.

\section{Литература/References}

1. C. Soares. Gas Turbines A Handbook of Air, Land and Sea Applications. Oxford, Butterworth-Heinemann, (2015).

2. A. V. Logunov, Yu. N. Shmotin. Modern high-temperature nickel-based alloys for gas turbine. Moscow, Nauka i tekhnologiya (2013) 256 p. (in Russian) [А. В. Логунов, Ю.Н. Шмотин. Современные жаропрочные никелевые сплавы для дисков газовых турбин. Москва, Наука и технология (2013) 256 с.]

3. M.M. Bakradze, A.V. Skugorev, V.V. Kucheryaev, M.V. Bubnov. Aviation Materials and Technologies. 5, 175 (2017). (in Russian) [M. M. Бакрадзе, А. В. Скугорев В. В. Кучеряев, М. В. Бубнов. Авиационные материалы и технологии. 5, 175 (2017). $\underline{\text { Crossref }}$

4. A. V. Lyushinsky. Svarochnoye proizvodstvo. 7, 17 (2016). (in Russian) [А.В. Люшинский. Сварочное производство. 7, 17 (2016).]

5. A. V. Skugorev, A. N. Afanasiev-Khodykin, A. M. Rogalev, D.S. Lozhkova. Tekhnologiya legkikh splavov. 3, 75 (2016). (in Russian) [А. В. Скугоров, А.Н. АфанасьевХодыкин, А. М. Рогалев, Д.С. Ложкова. Технология легких сплавов. 3, 75 (2016).]

6. I. Dolezel, V. Kotlan, B. Ulrych. Journal of Computational and Applied Mathematics. 270, 52 (2014). Crossref

7. J. Liu, J. Cao, X. Lin, X. Song, J. Feng. Materials and Design. 49, 622 (2013). $\underline{\text { Crossref }}$ 
8. O.G. Ospennikova, V.I. Lukin, A.N. AfanasevKhodykin, I.A. Galushka. Trudy VIAM. 10 (70), 10 (2018). (in Russian) [О.Г. Оспенникова, В.И. Лукин, А.Н. Афанасьев-Ходыкин, И.А. Галушка. Труды ВИАМ. 10 (70), 10 (2018).] Crossref

9. Z. Heng, M. Maeda, Y. Takahashi. IOP Conference Series: Materials Science and Engineering. 61 (1), 012003 (2014). Crossref

10. R. R. Mulyukov, A.A. Nazarov, R. M. Imayev. Letters on Materials. 8 (4s), 510 (2018). Crossref

11. E. V. Galieva, R. YA. Lutfullin, A. KH. Akhunova, V.A. Valitov, S. V. Dmitriev. Science and technology of welding and joining. 23 (7), 612 (2018). Crossref

12. E. V. Galieva, V. A. Valitov, R. Ya. Lutfullin, S. V. Dmitriev, A. Kh. Akhunova, M. Kh. Mukhametrakhimov. Materials Science Forum. 838-839, 350 (2016). Crossref

13. K. B. Povarova, A. A. Drozdov, V. A. Valitov, E. V. Valitova,
S. V. Obsepyan, O.A. Bazyleva. Russian metallurgy (Metally). 2014 (9), 733 (2014). $\underline{\text { Crossref }}$

14. A. K. Akhunova, E. V. Valitova, S. V. Dmitriev, V. A. Valitov, R. Y. Lutfullin. Welding International. 30 (6), 488 (2016). Crossref

15. V.A. Valitov, A. K. Akhunova, E. V. Galieva, S. V. Dmitriev, R. Y. Lutfullin, M. Y. Zhigalova. Lett. Mater. 7 (2), 180 (2017). (in Russian) [В.А. Валитов, А.Х. Ахунова, Э.В. Галиева, С.В. Дмитриев, Р.Я. Лутфуллин, М.Ю. Жигалова. Письма о материалах. 7 (2), 180 (2017).] Crossref

16. A.K. Akhunova, E.V. Galieva, A.A. Drozdov, E. G. Arginbaeva, S. V. Dmitriev, R. Y. Lutfullin. Lett. Mater. 6 (3), 211 (2016). (in Russian) [A.Х. Ахунова, Э.В. Галиева, А.А. Дроздов, Э.Г. Аргинбаева, С. В. Дмитриев, Р. Я. Лутфуллин. Письма о материалах. 6 (3), 211 (2016).] Crossref 